\title{
Hölder Continuity of Spectral Measures for the Finitely Differentiable Quasi-Periodic Schrödinger Operators
}

\author{
Mei Sun and Xueyin Wang* \\ Institute of Applied System Analysis, Jiangsu University, Zhenjiang, Jiangsu 212013, \\ China
}

Received 12 November 2019; Accepted (in revised version) 10 April 2020

\begin{abstract}
In the present paper, we prove the $\frac{1}{2}$-Hölder continuity of spectral measures for the $C^{k}$ Schrödinger operators. This result is based on the quantitative almost reducibility and an estimate for the growth of the Schrödinger cocycles in [5].
\end{abstract}

Key Words: Schrödinger operator, quasi-periodic, almost reducibility, finitely differentiable.

AMS Subject Classifications: 52B10, 65D18, 68U05, 68U07

\section{Introduction}

In this paper, we consider the Schrödinger operators defined on $\ell^{2}(\mathbb{Z})$

$$
\left(H_{V, \alpha, \theta} u\right)_{n}=u_{n+1}+u_{n-1}+V(\theta+n \alpha) u_{n}
$$

where $V: \mathbb{T}^{d} \rightarrow \mathbb{R}$ is the potential, $\theta \in \mathbb{T}^{d}=(\mathbb{R} / \mathbb{Z})^{d}$ is the phase, and $\alpha \in \mathbb{T}^{d}$ is the frequency.

These operators have been extensively and thoroughly studied for the deep connection with quasi-crystal and quantum Hall effects $[11,18]$. This paper concerns the regularity of the spectral measure of the quasi-periodic Schrödinger operators. For the analytic potential $V \in C^{\omega}\left(\mathbb{T}^{d}, \mathbb{R}\right)$, there is some significant progress $[5,21,24]$. However for the smooth potential $V \in C^{k}\left(\mathbb{T}^{d}, \mathbb{R}\right)$, there is no similar result as far as we know, so we will give a supplementary answer to this situation.

Let us review some results on the Hölder continuity of the integrated density of states (IDS) and the individual spectral measures.

*Corresponding author. Email addresses: sunm@ujs.edu . cn (M. Sun), 2889190407@qq. com (X. Wang) 


\subsection{Hölder continuity of IDS}

Let $\Sigma_{V, \alpha, \theta}$ be the spectrum of $H_{V, \alpha, \theta}$, then $\Sigma_{V, \alpha, \theta} \subset \mathbb{R}$ since $H_{V, \alpha, \theta}$ is the bounded selfadjoint operator in $\ell^{2}(\mathbb{Z})$. The spectrum is independent of $\theta$ if $(\alpha, 1)$ is rational independent. For any $f \in \ell^{2}(\mathbb{Z})$, the spectral measure $\mu_{V, \alpha, \theta}^{f}$ of $H_{V, \alpha, \theta}$ can be defined as

$$
\left\langle\left(H_{V, \alpha, \theta}-E\right)^{-1} f, f\right\rangle=\int_{\mathbb{R}} \frac{1}{E^{\prime}-E} d \mu_{V, \alpha, \theta}^{f}\left(E^{\prime}\right), \quad \forall E \in \mathbb{C} \backslash \Sigma_{V, \alpha} .
$$

Let $\mu_{V, \alpha, \theta}=\mu_{V, \alpha, \theta}^{e_{-1}}+\mu_{V, \alpha, \theta}^{e_{0}}$, where $\left\{e_{i}\right\}_{i \in \mathbb{Z}}$ is the cannonical basis of $\ell^{2}(\mathbb{Z})$. Let $N_{V, \alpha}$ be the IDS of $H_{V, \alpha, \theta}$, it is well known that IDS is the average of the spectral measure $\mu_{V, \alpha, \theta}$ with respect to $\theta$, i.e.,

$$
N_{V, \alpha}(E)=\int_{\mathbb{T}^{d}} \mu_{V, \alpha, \theta}(-\infty, E] d \theta .
$$

Hence the regularity of IDS is closely related to that of the spectral measure.

Recall that $\alpha \in \mathbb{T}^{d}$ is Diophantine if there exist $\gamma>0$ and $\tau>d-1$ such that $\alpha \in$ $\mathrm{DC}_{d}(\gamma, \tau)$, where

$$
\mathrm{DC}_{d}(\gamma, \tau)=\left\{\alpha: \inf _{j \in \mathbb{Z}}|\langle n, \alpha\rangle-j|>\frac{\gamma}{|n|^{\tau}}, \forall n \in \mathbb{Z}^{d} \backslash\{0\}\right\} .
$$

Let $\mathrm{DC}_{d}=\cup_{\gamma>0, \tau>d-1} \mathrm{DC}_{d}(\gamma, \tau)$. For $\alpha \in \mathbb{R} \backslash \mathbb{Q}$, let $\frac{p_{n}}{q_{n}}$ be the continued fraction approximants to $\alpha$, then one can define

$$
\beta(\alpha)=\limsup _{n \rightarrow \infty} \frac{\ln q_{n+1}}{q_{n}} .
$$

Given the operator $H_{V, \alpha, \theta}$, one can define the Lyapunov exponent $L\left(\alpha, S_{E}^{V}\right)$ (see Section 2.1) of the corresponding Schrödinger cocycle $\left(\alpha, S_{E}^{V}(\theta)\right)$, where $E \in \mathbb{R}$ and

$$
S_{E}^{V}(\theta)=\left(\begin{array}{cc}
E-V(\theta) & -1 \\
1 & 0
\end{array}\right)
$$

Hadj Amor [16] proved the $\frac{1}{2}$-Hölder continuity of IDS if $\alpha \in \mathrm{DC}_{d}$ and $V \in C^{\omega}\left(\mathbb{T}^{d}, \mathbb{R}\right)$ is small, and her approach is based on the almost reducibility scheme developed by Eliasson [13]. Recall that the cocycle $(\alpha, A)$ is reducible if $(\alpha, A)$ can be conjugated to some constant cocycles and the cocycle $(\alpha, A)$ is almost reducible if the closure of its conjugates contains a constant. Avila and Jitomirskaya [4] proved the $\frac{1}{2}$-Hölder continuity of IDS for $\alpha \in \mathrm{DC}_{1}$ and the small analytic potential. Their result was non-perturbative, which means that the smallness is independent of $\alpha$. After that, Avila [2,3] generalized the result for the small analytic potential with $\beta(\alpha)=0$ if there is $\delta>0$ such that $L\left(\alpha, S_{E+i \epsilon}^{V}\right)=0$ for $|\epsilon|<\delta$. Note that Leguil-You-Zhao-Zhou [20] showed the same result as well by the global theory of the one-frequency Schrödinger operators [1]. 
The Hölder continuity of $N_{V, \alpha}(E)$ is equivalent to the Hölder continuity of $L\left(\alpha, S_{E}^{V}\right)$ according to the famous Thouless formula [6], i.e.,

$$
L\left(\alpha, S_{E}^{V}\right)=\int \ln \left|E-E^{\prime}\right| d N_{V, \alpha}\left(E^{\prime}\right) .
$$

Suppose that $L\left(\alpha, S_{E}^{V}\right)>0$. Goldstein and Schlag [14] proved the Hölder continuity of $L\left(\alpha, S_{E}^{V}\right)$ if $V \in C^{\omega}(\mathbb{T}, \mathbb{R})$, and $\alpha \in S D C^{\dagger}$ by the avalanche principle and sharp large deviation theorem. Later, You and Zhang [22] proved that for analytic potential, the $L\left(\alpha, S_{E}^{V}\right)$ is Hölder continuous if $\alpha \in \mathrm{DC}_{1}$ or some weaker Liovillean $\alpha$ by a refined large deviation theorem.

For the special kinds of potentials, there are some other interesting results. If the potential is a small perturbation of a trigonometric polynomial of degree $d$ and $\alpha \in \mathrm{DC}_{1}$, Goldetein-Schlag [15] proved that IDS is $\left(\frac{1}{2 d}-\epsilon\right)$-Hölder continuous for any $\epsilon>0$ by assuming $L\left(\alpha, S_{E}^{V}\right)>0$. In particular, if the potential $V(x)=2 \lambda \cos (x)$, we get the so called almost Mathieu operators (AMO), i.e.,

$$
\left(H_{\lambda, \alpha, \theta} u\right)_{n}=u_{n+1}+u_{n-1}+2 \lambda \cos (\theta+n \alpha) u_{n}
$$

where $\lambda \in \mathbb{R}$ is the coupling constant. For the large coupling, Bourgain [7] proved that Lyapunov exponent of AMO is $\left(\frac{1}{2}-\epsilon\right)$-Hölder continuous for any $\epsilon>0$. Avila [2] obtained the exact $\frac{1}{2}$-Hölder continuity of IDS for AMO with $|\lambda|<1$.

\subsection{Hölder continuity of the spectral measure}

In general, the spectral measure is less regular than IDS. In the $\beta(\alpha)=0$ regime, Avila and Jitomirskaya [5] proved that if $V \in C^{\omega}(\mathbb{T}, \mathbb{R})$ is small enough and $\alpha \in \mathrm{DC}_{1}$, then the spectral measure $\mu_{V, \alpha, \theta}^{f}$ of one-frequency Schrödinger operators is $\frac{1}{2}$-Hölder continuous for any $f \in \ell^{1}(\mathbb{Z}) \cap \ell^{2}(\mathbb{Z})$. They also showed $\frac{1}{2}$-Hölder continuity of absolutely continuous spectral measures for the one-frequency Schrödinger operators with $V \in C^{\omega}(\mathbb{T}, \mathbb{R})$. In the $\beta(\alpha)>0$ regime, Liu and Yuan [21] extended the results in [5] to that for all $\alpha \in \mathbb{R} \backslash \mathbb{Q}$ with $\beta(\alpha)<\infty$, whenever the analytic radius of $V$ is sufficiently large.

What can we say about the regularity of the spectral measure of Schrödinger operators with finitely differentiable potential? Recall that Cai-Chavaudret-You-Zhou [10] have shown that if $\alpha \in \mathrm{DC}_{d}$ and $V \in C^{k}\left(\mathbb{T}^{d}, \mathbb{R}\right)$ is small, then IDS of the Schrödinger operator is $\frac{1}{2}$-Hölder continuous. Recently, Zhao [24] also generalized [5] and [21] to the multi-frequency Schrödinger operators with $V \in C^{\omega}\left(\mathbb{T}^{d}, \mathbb{R}\right)$. This paper is motivated by [10] and [24], as a supplementray answer, we obtain the $\frac{1}{2}$-Hölder continuity of the spectral measure $\mu_{V, \alpha, \theta}^{f}$ for $V \in C^{k}\left(\mathbb{T}^{d}, \mathbb{R}\right)$.

\footnotetext{
${ }^{\dagger}$ We say $\alpha$ satifies strong Diophantine condition if there exist some $\gamma>0, \tau>1$ such that $\alpha \in S D C(\gamma, \tau)$, where

$$
\operatorname{SDC}(\gamma, \tau)=\left\{\alpha \in \mathbb{R}^{d}: \inf _{j \in \mathbb{Z}}|\langle n, \alpha\rangle-j|>\frac{\gamma}{|n|(\ln (1+|n|))^{\tau}}\right\},
$$


Theorem 1.1. Let $\alpha \in \mathrm{DC}_{d}(\gamma, \tau), V \in C^{k}\left(\mathbb{T}^{d}, \mathbb{R}\right)$ with $k \geq 5 D \tau$ and $D$ is a numerical constant. There exists $\tilde{\varepsilon}=\tilde{\varepsilon}(\gamma, \tau, k)$ such that if $\|V\|_{k} \leq \tilde{\varepsilon}$, then for any $f \in \ell^{2}(\mathbb{Z}) \cap \ell^{1}(\mathbb{Z})$,

$$
\mu_{V, \alpha, \theta}^{f}(J) \leq D_{0}|J|^{\frac{1}{2}}\|f\|_{\ell^{1}}^{2}
$$

for all intervals $J$ and all $\theta$, where $D_{0}=D_{0}(V, \alpha)>0$.

Remark 1.1. Note that Theorem 1.1 is perturbative, i.e., the smallness $\tilde{\varepsilon}$ depends not only on the potential $V$, but also on the frequency $\alpha$. From a counterexample of Bourgain [8], one can not expect non-perturbative results in multi-frequency case.

\section{Preliminaries}

For a bounded analytic function $F$ defined on $\mathcal{S}_{r}=\left\{\theta: \theta=\left(\theta_{1}, \cdots, \theta_{d}\right) \in \mathbb{C}^{d},\left|\Im \theta_{i}\right|<\right.$ $r, \forall i=1, \cdots, d\}$, let $|F|_{r}=\sup _{\theta \in \mathcal{S}_{r}}\|F(\theta)\|$ and denote by $C_{r}^{\omega}\left(\mathbb{T}^{d}, *\right)$ the set of these $*-$ value functions ( $*$ will usually denote $\mathbb{R}, \operatorname{sl}(2, \mathbb{R})$ or $S L(2, \mathbb{R})$ ). We also denote the set $C^{k}\left(\mathbb{T}^{d}, *\right)$ to be the space of $k$ times differentiable with continuous $k$-th derivatives functions, endowed with the norm

$$
\|F\|_{k}:=\sup _{k^{\prime} \leq k, \theta \in \mathbb{T}^{d}}\left\|\partial^{k^{\prime}} F(\theta)\right\|
$$

In particular,

$$
\|F\|_{0}:=\|F\|_{\mathbb{T}^{d}}=\sup _{\theta \in \mathbb{T}^{d}}\|F(\theta)\| .
$$

For $\theta \in \mathbb{R}$, we set $\|\theta\|_{\mathbb{T}}=\inf _{j \in \mathbb{Z}}|\theta-j|$.

\subsection{Uniform hyperbolicity}

Given $A \in C^{\omega}\left(\mathbb{T}^{d}, S L(2, \mathbb{C})\right)$ and $\alpha \in \mathbb{R}^{d}$ rationally independent, one can define the quasi-periodic (Q-P) cocycle $(\alpha, A)$ :

$$
\begin{aligned}
(\alpha, A): & \mathbb{T}^{d} \times \mathbb{C}^{2} \rightarrow \mathbb{T}^{d} \times \mathbb{C}^{2} ; \\
(\theta, v) & \mapsto(\theta+\alpha, A(\theta) \cdot v) .
\end{aligned}
$$

The iterations of $(\alpha, A)$ are of form $(\alpha, A)^{n}=\left(n \alpha, A_{n}\right)$, where

$$
A_{n}(\theta):= \begin{cases}A(\theta+(n-1) \alpha) \cdots A(\theta+\alpha) A(\theta), & n \geq 0, \\ A^{-1}(\theta+n \alpha) A^{-1}(\theta+(n+1) \alpha) \cdots A^{-1}(\theta-\alpha), & n<0 .\end{cases}
$$

The Lyapunov exponent of the cocycle $(\alpha, A)$ is defined as

$$
L(\alpha, A)=\lim _{n \rightarrow \infty} \frac{1}{n} \int_{\mathbb{T}^{d}}\left\|A_{n}(\theta)\right\| d \theta .
$$


We say the cocycle $(\alpha, A)$ is uniformly hyperbolic if for every $\theta \in \mathbb{T}^{d}$, there exists a continuous splitting $\mathbb{C}^{2}=E^{s}(\theta) \oplus E^{u}(\theta)$ such that for every $n \geq 0$,

$$
\begin{array}{ll}
\left\|A_{n}(\theta) v\right\| \leq C e^{-c n}\|v\|, & v \in E^{S}(\theta), \\
\left\|A_{n}(\theta)^{-1} v\right\| \leq C e^{-c n}\|v\|, & v \in E^{u}(\theta+n \alpha),
\end{array}
$$

for some constants $C, c>0$. And the splitting is invariant by the dynamics:

$$
\begin{array}{ll}
A(\theta) E^{s}(\theta)=E^{s}(\theta+\alpha), & \forall \theta \in \mathbb{T}^{d}, \\
A(\theta) E^{u}(\theta)=E^{u}(\theta+\alpha), & \forall \theta \in \mathbb{T}^{d} .
\end{array}
$$

\subsection{Spectral measure and Wely's m function}

Typical examples of $S L(2, \mathbb{R})$ cocycles are the Schrödinger cocycles $\left(\alpha, S_{E}^{V}\right)$ :

$$
A(\theta)=S_{E}^{V}(\theta)=\left(\begin{array}{cc}
E-V(\theta) & -1 \\
1 & 0
\end{array}\right), \quad E \in \mathbb{R} .
$$

Those cocycles come from the eigenvalue equation of one dimensional quasi-periodic Schrödinger operators on $\ell^{2}(\mathbb{Z})$ :

$$
\left(H_{V, \alpha, \theta} u\right)_{n}=u_{n+1}+u_{n-1}+V(\theta+n \alpha) u_{n}=E u_{n},
$$

and any formal solution $u=\left(u_{n}\right)_{n \in \mathbb{Z}}$ of $H_{V, \alpha, \theta} u=E u$ satisfies

$$
\left(\begin{array}{c}
u_{n+1} \\
u_{n}
\end{array}\right)=S_{E}^{V}(\theta+n \alpha)\left(\begin{array}{c}
u_{n} \\
u_{n-1}
\end{array}\right), \quad \forall n \in \mathbb{Z} .
$$

For any $f \in \ell^{2}(\mathbb{Z})$, one can define the spectral measure $\mu_{V, \alpha, \theta}^{f}$ corresponding to $f$ as in (1.1). Given $E+i \epsilon$ with $E \in \mathbb{R}$ and $\epsilon>0$, there exists a non-zero solution $u^{+}$of $H_{V, \alpha, \theta} u^{+}=(E+i \epsilon) u^{+}$being square-summable at $+\infty$. The Wely's $m$ function is given by $m^{+}=-\frac{u_{1}^{+}}{u_{0}^{+}}$.

Let

$$
M(E+i \epsilon)=\int_{\mathbb{R}} \frac{1}{E^{\prime}-(E+i \epsilon)} d \mu_{V, \alpha, \theta}\left(E^{\prime}\right) .
$$

From the definition of $M(\cdot)$, it deduces immediately that $M(\cdot)$ is a Herglotz function defined on $\mathbb{H}=\{z: \Im z>0\}$, and

$$
\Im M(E+i \epsilon) \geq \frac{1}{2 \epsilon} \mu_{V, \alpha, \theta}(E-\epsilon, E+\epsilon) .
$$

Recall that in [12], by the usual action of $S L(2, \mathbb{C})$, we denote

$$
m_{\beta}^{+}:=R_{-\frac{\beta}{2 \pi}} \cdot m^{+}=\frac{m^{+} \cos \beta-\sin \beta}{m^{+} \sin \beta+\cos \beta},
$$

then we have the following lemma: 
Lemma 2.1 ([5]). Let $\psi\left(m^{+}\right):=\sup _{\beta}\left|m_{\beta}^{+}\right|$, then $\|M\|_{0} \leq \psi\left(m^{+}\right)$.

The spectral properties of $H_{V, \alpha, \theta}$ and the dynamics of $\left(\alpha, S_{E}^{V}\right)$ are closely related by the fact: $E \in \Sigma_{V, \alpha}$ if and only if $\left(\alpha, S_{E}^{V}\right)$ is not uniformly hyperbolic [19].

\subsection{Analytic approximation}

Assume $f \in C^{k}\left(\mathbb{T}^{d}, s l(2, \mathbb{R})\right)$, according to [23], there exists a sequence $\left\{f_{j}\right\}_{j \geq 1}$ with $f_{j} \in$ $C_{\frac{1}{j}}^{\omega}\left(\mathbb{T}^{d}, s l(2, \mathbb{R})\right)$ and a universal constant $C^{\prime}>0$, such that

$$
\begin{aligned}
& \left\|f_{j}-f\right\|_{k} \rightarrow 0, \quad j \rightarrow+\infty \\
& \left|f_{j}\right|_{\frac{1}{j}} \leq C^{\prime}\|f\|_{k} \\
& \left|f_{j+1}-f_{j}\right|_{\frac{1}{j+1}} \leq C^{\prime}(j)^{-k}\|f\|_{k} .
\end{aligned}
$$

\subsection{Space decomposition}

Given $A \in S L(2, \mathbb{R}), \alpha \in \mathbb{R}^{d}$ and $\eta>0$, one can obtain the decomposition $\mathcal{B}_{k}=$ $\mathcal{B}_{k}^{(n r e)}(\eta) \oplus \mathcal{B}_{k}^{(r e)}(\eta)$, where

$$
\mathcal{B}_{k}=\left\{f \in C^{k}\left(\mathbb{T}^{d}, s l(2, \mathbb{R})\right):\|f\|_{k}<\infty\right\},
$$

and $\mathcal{B}_{k}^{(\text {nre })}(\eta)$ is the subspace of $\mathcal{B}_{k}$ such that for any $Y \in \mathcal{B}_{k}^{(\text {nre })}(\eta)$,

$$
A^{-1} Y(\theta+\alpha) A \in \mathcal{B}_{k}^{(n r e)}(\eta), \quad\left\|A^{-1} Y(\theta+\alpha) A-Y(\theta)\right\|_{k}>\eta\|Y\|_{k} .
$$

Lemma 2.2 ([10,17]). Assume that $A \in S L(2, \mathbb{R}), \alpha \in \mathbb{R}^{d}, \varepsilon^{\prime} \leq(4\|A\|)^{-4}$, and $\eta \geq$ $13\|A\|^{2} \mathcal{E}^{\prime \frac{1}{2}}$. For any $g \in \mathcal{B}_{k}$ with $\|g\|_{k} \leq \mathcal{E}^{\prime}$, there exist $Y \in \mathcal{B}_{k}$ and $g^{\text {re }} \in \mathcal{B}_{k}^{r e}(\eta)$ such that

$$
e^{-Y(\theta+\alpha)} A e^{g(\theta)} e^{Y(\theta)}=A e^{g^{r e}(\theta)},
$$

with $\|Y\|_{k} \leq \varepsilon^{\prime \frac{1}{2}}$, and $\left\|g^{r e}\right\|_{k} \leq 2 \varepsilon^{\prime}$.

The continuous version ( $C^{\omega}$ linear systems) and discrete version $\left(C^{\omega}\right.$ cocyles) of Lemma 2.2 are shown in [17] and [10] respectively. The proof of Lemma 2.2 only depends on $\mathcal{B}_{k}$ being a Banach space, thus one can obtain the result by replacing analytic norm with $C^{k}$ norm in the Appendix of [10].

\section{Dynamical estimates of $C^{k}$ quasi-periodic cocycles}

To deal with the almost reducibility of the $C^{k}$ cocycles, the strategy used here is treating the analytic cocycles firstly and turning the estimates of analytic cocycles into those of finitely differentiable cocycles by analytic approximation. 
Consider the following Q-P $S L(2, \mathbb{R})$ cocycle

$$
\begin{aligned}
\left(\alpha, A e^{f(\theta)}\right): & \mathbb{T}^{d} \times \mathbb{R}^{2} \rightarrow \mathbb{T}^{d} \times \mathbb{R}^{2} ; \\
(\theta, x) & \mapsto\left(\theta+\alpha, A e^{f(\theta)} \cdot x\right),
\end{aligned}
$$

where $\alpha \in \operatorname{DC}_{d}(\gamma, \tau), A \in S L(2, \mathbb{R})$ and $f(\theta) \in C_{r}^{\omega}\left(\mathbb{T}^{d}, s l(2, \mathbb{R})\right)$ with $r>0$. Assume $|f|_{r} \leq \varepsilon$, we are going to show that the perturbation will tend to zero by KAM scheme.

Proposition 3.1 ([10,20]). Let $\alpha \in \mathrm{DC}_{d}(\gamma, \tau), \gamma, \gamma>0, \tau>d-1$ and $\sigma=\frac{1}{10}$. Then for any $r_{+} \in(0, r)$, there exist $c=c(\gamma, \tau, d)$ and a numerical constant $D$ such that if

$$
\varepsilon \leq \frac{c}{\|A\|^{D}}\left(r-r_{+}\right)^{D \tau}
$$

then there exist $B(\theta) \in C_{r_{+}}^{\omega}\left(2 \mathbb{T}^{d}, S L(2, \mathbb{R})\right), A_{+} \in S L(2, \mathbb{R})$ and $f_{+}(\theta) \in C_{r_{+}}^{\omega}\left(\mathbb{T}^{d}, s l(2, \mathbb{R})\right)$ such that $\left(\alpha, A e^{f(\theta)}\right)$ is conjugated to $\left(\alpha, A_{+} e^{f_{+}(\theta)}\right)$ by $B(\theta)$, i.e.,

$$
B(\theta+\alpha)^{-1} A e^{f(\theta)} B(\theta)=A_{+} e^{f_{+}(\theta)} .
$$

More precisely, let $N=\frac{2|\ln \varepsilon|}{r-r_{+}}$and $\left\{e^{2 \pi i \rho}, e^{-2 \pi i \rho}\right\}$ be the two eigenvalues of $A$, we can distinguish between two cases:

(A) (Non-resonant case). Assume that

$$
\|2 \rho-\langle n, \alpha\rangle\|_{\mathbb{T}} \geq \varepsilon^{\sigma}, \quad \forall n \in \mathbb{Z}^{d} \text { with } 0<|n| \leq N,
$$

then we have the etsimates:

$$
\left|f_{+}(\theta)\right|_{r_{+}} \leq 4 \varepsilon^{3-2 \sigma}, \quad|B(\theta)-I d|_{r_{+}} \leq \varepsilon^{\frac{1}{2}}, \quad\left\|A_{+}-A\right\| \leq 2\|A\| \varepsilon .
$$

(B) (Resonant case). If there exists $n^{*} \in \mathbb{Z}^{d}$ with $0<\left|n^{*}\right| \leq N$ such that

$$
\left\|2 \rho-\left\langle n^{*}, \alpha\right\rangle\right\|_{\mathbb{T}}<\varepsilon^{\sigma},
$$

then we have the estimates:

$$
|B(\theta)|_{r_{+}} \leq C_{1}\left|n^{*}\right|^{\frac{\tau}{2}} e^{\pi\left|n^{*}\right| r_{+}}, \quad\|B(\theta)\|_{0} \leq C_{1}\left|n^{*}\right|^{\frac{\tau}{2}}, \quad\left|f_{+}(\theta)\right|_{r_{+}} \ll \varepsilon^{100},
$$

where $C_{1}=4\|A\|^{\frac{1}{2}} \gamma^{-\frac{1}{2}}$. Moreover, let $A_{+}:=e^{A^{\prime \prime}}$ with $A^{\prime \prime} \in \operatorname{sl}(2, \mathbb{R})$, then $\left\|A^{\prime \prime}\right\| \leq 16 \varepsilon^{\sigma}$.

\subsection{Real quantitative estimates for $C^{k} Q-P$ cocycles}

We are going to deal with the almost reducibility of the $C^{k}$ cocycles by analytic approximation. Let $\left\{f_{j}\right\}_{j \geq 1}, f_{j} \in C_{\frac{1}{j}}^{\omega}\left(\mathbb{T}^{d}, s l(2, \mathbb{R})\right)$ be the analytic sequence approximating 
$f \in C^{k}\left(\mathbb{T}^{d}, s l(2, \mathbb{R})\right)$. We first recall some notations given in [10]. $D$ is a numerical constant defined by Proposition 3.1, we denote

$$
\varepsilon_{0}^{\prime}\left(h, h^{\prime}\right):=\frac{c}{(2\|A\|)^{D}}\left(h-h^{\prime}\right)^{D \tau},
$$

and define

$$
\varepsilon_{m}:=\frac{c}{(2\|A\|)^{D^{\frac{k}{4}}}} .
$$

Then one can check that for any $k \geq 5 D \tau$ and any $m \geq 10, m \in \mathbb{Z}$,

$$
\frac{c}{(2\|A\|)^{D_{m^{\frac{k}{4}}}}} \leq \varepsilon_{0}^{\prime}\left(\frac{1}{m^{\prime}}, \frac{1}{m^{2}}\right) .
$$

Denote $l_{j}=M^{2^{j-1}}, \forall j \in \mathbb{Z}^{+}$, where $M>\max \left\{10, \frac{(2\|A\|)^{D}}{c}\right\}$ is an integer.

Theorem 3.1. Let $\alpha \in \mathrm{DC}_{d}(\gamma, \tau), A \in S L(2, \mathbb{R}), \sigma=\frac{1}{10}, f(\theta) \in C^{k}\left(\mathbb{T}^{d}, s l(2, \mathbb{R})\right)$ with $k \geq 5 D \tau$. Let $\left\{f_{j}\right\}_{j \geq 1}$ be the analytic sequence approximating $f(\theta)$ defined in (2.2). There exists $\bar{\varepsilon}=\bar{\varepsilon}(\gamma, \tau, d, k,\|A\|)$ such that if $\|f\|_{k} \leq \bar{\varepsilon}$, then the following holds:

(A) There exist $B_{l_{j}}(\theta) \in C_{\frac{1}{l_{j+1}}}^{\omega}\left(2 \mathbb{T}^{d}, S L(2, \mathbb{R})\right), A_{l_{j}} \in S L(2, \mathbb{R})$ and $f_{l_{j}}^{\prime}(\theta) \in C_{\frac{1}{l_{j+1}}}^{\omega}\left(\mathbb{T}^{d}, s l(2, \mathbb{R})\right)$ such that

$$
B_{l_{j}}(\theta+\alpha)^{-1} A e^{f_{l_{j}}(\theta)} B_{l_{j}}(\theta)=A_{l_{j}} e^{f_{l_{j}}^{\prime}(\theta)},
$$

with following estimates

$$
\begin{aligned}
& \left\|B_{l_{j}}(\theta)\right\|_{0} \leq\left(l_{j}\left|\ln \varepsilon_{l_{j}}\right|\right)^{\tau(1+\sigma)}, \quad\left|f_{l_{j}}^{\prime}(\theta)\right|_{\frac{1}{l_{j+1}}} \leq \frac{1}{2} \varepsilon_{l_{j_{j}}}^{\frac{5}{2}}, \\
& \left|B_{l_{j}}(\theta)\right|_{\frac{1}{l_{j+1}}} \leq\left(l_{j}\left|\ln \varepsilon_{l_{j}}\right|\right)^{\tau(1+\sigma)} \varepsilon_{l_{j}}^{-\xi},
\end{aligned}
$$

where $\xi \in\left(\frac{2 \pi}{M^{2}-1}, \frac{1}{2}\right)$ is a constant.

(B) Moreover, $\left\|A_{l_{j}}\right\| \leq 2\|A\|$ and there exist some unitary matrices $U_{j} \in S L(2, \mathbb{C})$ such that $A_{l_{j}}$ can be written as

$$
A_{l_{j}}=U_{j}\left(\begin{array}{cc}
e^{2 \pi i \rho_{j}} & c_{j} \\
0 & e^{-2 \pi i \rho_{j}}
\end{array}\right) U_{j}^{-1} \text { with } \rho_{j} \in i \mathbb{R} \cup \mathbb{R} \text { and } c_{j} \in \mathbb{C} .
$$

Then for any constant $\kappa \in\left[1, \frac{\sigma k}{8 \tau}\right)$, there exists constant $C=C(\|A\|)>0$ such that

$$
\left\|B_{l_{j}}(\theta)\right\|_{0}^{\kappa} \cdot\left|c_{j}\right| \leq C
$$

Proof. We will prove Theorem 3.1 by the induction process as in [9] and [10].

First Step. Suppose that

$$
\|f\|_{k} \leq \frac{c}{C^{\prime}(2\|A\|)^{D} M^{\frac{k}{4}}}
$$


where $C^{\prime}$ is a universal constant defined in (2.2). Thus we have

$$
\left|f_{l_{1}}\right|_{\frac{1}{l_{1}}} \leq \varepsilon_{l_{1}} \leq \varepsilon_{0}^{\prime}\left(\frac{1}{l_{1}}, \frac{1}{l_{2}}\right)
$$

By Proposition 3.1, one can find $B_{l_{1}}(\theta) \in C_{\frac{1}{l_{2}}}^{\omega}\left(2 \mathbb{T}^{d}, S L(2, \mathbb{R})\right), A_{l_{1}} \in S L(2, \mathbb{R})$ and $f_{l_{1}}^{\prime}(\theta) \in$ $C_{\frac{1}{l_{2}}}^{\omega}\left(\mathbb{T}^{d}, \operatorname{sl}(2, \mathbb{R})\right)$ such that

$$
B_{l_{1}}(\theta+\alpha)^{-1} A e^{f_{l_{1}}(\theta)} B_{l_{1}}(\theta)=A_{l_{1}} e^{f_{l_{1}}^{\prime}(\theta)} .
$$

More precisely, let $N_{l_{1}}=\frac{2\left|\ln \varepsilon_{l_{1}}\right|}{\frac{1}{l_{1}}-\frac{1}{l_{2}}}$ and $\left\{e^{2 \pi i \rho}, e^{-2 \pi i \rho}\right\}$ be two eigenvalues of $A$, we can distunguish two cases:

(Non-resonant case). If the first step is obtained by non-resonant case:

$$
\|2 \rho-\langle n, \alpha\rangle\|_{\mathbb{T}} \geq \varepsilon_{l_{1}}^{\sigma}, \quad \forall n \in \mathbb{Z}^{d} \quad \text { with } 0<|n| \leq N_{l_{1}},
$$

then we have the etsimates:

$$
\left|f_{l_{1}}^{\prime}(\theta)\right|_{\frac{1}{l_{2}}} \leq 4 \varepsilon_{l_{1}}^{3-2 \sigma}, \quad\left|B_{l_{1}}(\theta)\right|_{\frac{1}{l_{2}}} \leq 1+\varepsilon_{l_{1}}^{\frac{1}{2}}, \quad\left\|A_{l_{1}}-A\right\| \leq 2\|A\| \varepsilon_{l_{1}} .
$$

(Resonant case). If the first step is obtained by resonant case: there exists $n_{l_{1}}^{*} \in \mathbb{Z}^{d}$ with $0<\left|n_{l_{1}}^{*}\right| \leq N_{l_{1}}$ such that $\left\|2 \rho-\left\langle n_{l_{1}}^{*}, \alpha\right\rangle\right\|_{\mathbb{T}}<\varepsilon_{l_{1}}^{\sigma}$, then

$$
\begin{aligned}
\left\|B_{l_{1}}(\theta)\right\|_{0} \leq C_{2}(\gamma, \tau,\|A\|)\left(l_{1}\left|\ln \varepsilon_{l_{1}}\right|\right)^{\frac{\tau}{2}} \leq\left(l_{1}\left|\ln \varepsilon_{l_{1}}\right|\right)^{\tau(1+\sigma)}, \\
\left|B_{l_{1}}(\theta)\right|_{\frac{1}{l_{2}}} \leq\left(l_{1}\left|\ln \varepsilon_{l_{1}}\right|\right)^{\tau(1+\sigma)} \varepsilon_{l_{1}}^{\frac{-2 \pi}{2-1}}, \quad\left|f_{l_{1}}^{\prime}(\theta)\right|_{\frac{1}{l_{2}}} \ll \varepsilon_{l_{1}}^{100}<\frac{1}{2} \varepsilon_{l_{1}}^{\frac{5}{2}} .
\end{aligned}
$$

Moreover, let $A_{l_{1}}:=e^{A_{l_{1}}^{\prime \prime}}$ with $A_{l_{1}}^{\prime \prime} \in \operatorname{sl}(2, \mathbb{R})$ we have $\left\|A_{l_{1}}^{\prime \prime}\right\| \leq 16 \varepsilon_{l_{1}}^{\sigma}$.

Induction Step: Assume that in $\left(l_{j}\right)$-th step with $j \leq n$, we already have that

$$
B_{l_{j}}(\theta+\alpha)^{-1} A e^{f_{l_{j}}(\theta)} B_{l_{j}}(\theta)=A_{l_{j}} e^{f_{l_{j}}^{\prime}(\theta)}
$$

with the following estimates

$$
\begin{aligned}
& \left|B_{l_{j}}(\theta)\right|_{\frac{1}{l_{j+1}}} \leq\left(l_{j}\left|\ln \varepsilon_{l_{j}}\right|\right)^{\tau(\sigma+1)} \varepsilon_{l_{j}}^{-\tau}, \quad\left\|A_{l_{j}}\right\| \leq 2\|A\|, \\
& \left\|B_{l_{j}}\right\|_{0} \leq\left(l_{j}\left|\ln \varepsilon_{l_{j}}\right|\right)^{\tau(\sigma+1)}, \quad\left|f_{l_{j}}^{\prime}(\theta)\right|_{\frac{1}{l_{j+1}}} \leq \frac{1}{2} \varepsilon_{l_{j}}^{\frac{5}{2}} .
\end{aligned}
$$

Moreover, if the $\left(l_{j}\right)$-th step is obtained by the resontant case, we have

$$
A_{l_{j}}=e^{A_{l_{j}}^{\prime \prime}}, \quad\left\|A_{l_{j}}^{\prime \prime}\right\| \leq 8 \varepsilon_{l_{j^{\prime}}}^{\sigma}, \quad\left\|A_{l_{j}}\right\| \leq 1+16 \varepsilon_{l_{j}}^{\sigma} .
$$


If the $\left(l_{j}\right)$-th step is obtained by the non-resontant case, we have

$$
\left\|A_{l_{j}}-A_{l_{j-1}}\right\| \leq 2\left\|A_{l_{j-1}}\right\| \varepsilon_{l_{j}}, \quad\left|B_{l_{j}}\right|_{\frac{1}{l_{j+1}}} \leq\left(1+\varepsilon_{l_{j}}^{\frac{1}{2}}\right)\left|B_{l_{j-1}}\right|_{\frac{1}{l_{j}}} .
$$

Now let $j=n+1$ and focus on the cocycle $\left(\alpha, A e^{f_{l_{n+1}}(\theta)}\right)$, it follows that

$$
B_{l_{n}}(\theta+\alpha)^{-1} A e^{f_{l_{n+1}}} B_{l_{n}}(\theta)=A_{l_{n}} e^{f_{l_{n}}^{\prime}}+B_{l_{n}}(\theta+\alpha)^{-1}\left(A e^{f_{l_{n+1}}}-A e^{f_{l_{n}}}\right) B_{l_{n}}(\theta) .
$$

If we rewrite

$$
A_{l_{n}} e^{f_{l_{n}}^{\prime}}+B_{l_{n}}(\theta+\alpha)^{-1}\left(A e^{f_{l_{n+1}}}-A e^{f_{l_{n}}}\right) B_{l_{n}}(\theta)=A_{l_{n}} e^{\overline{f_{l_{n}}}(\theta)},
$$

by (3.3) and $\xi \in\left(\frac{2 \pi}{M^{2}-1}, \frac{1}{2}\right)$, we have

$$
\begin{aligned}
\left|\overline{f_{l_{n}}}(\theta)\right|_{\frac{1}{l_{n+1}}} & \leq\left|f_{l_{n}}^{\prime}\right|_{\frac{1}{l_{n+1}}}+\left\|A_{l_{n}}^{-1}\right\| \cdot\left|B_{l_{n}}(\theta+\alpha)^{-1}\left(A e^{f_{l_{n+1}}}-A e^{f_{l_{n}}}\right) B_{l_{n}}\right|_{\frac{1}{l_{n+1}}} \\
& \leq \frac{1}{2} \varepsilon_{l_{n}}^{\frac{5}{2}}+2\|A\|^{2} \times\left(l_{n}\left|\ln \varepsilon_{l_{n}}\right|\right)^{2 \tau(\sigma+1)} \varepsilon_{l_{n}}^{-2 \tau} \times \frac{c}{(2\|A\|)^{D} l_{n}^{k-1}} \\
& \leq \frac{1}{2} \varepsilon_{l_{n+1}}+\frac{1}{2} \times \frac{c}{(2\|A\|)^{D} l_{n}^{\frac{k}{2}}} \leq \varepsilon_{l_{n+1}} .
\end{aligned}
$$

Apply Proposition 3.1 to the cocycle $\left(\alpha, A_{l_{n}} e^{\overline{l_{n}}(\theta)}\right)$, one can obtain $\widetilde{B}_{l_{n}}(\theta) \in$ $\underset{\frac{1}{I_{n+2}}}{C_{1}^{\omega}}\left(2 \mathbb{T}^{d}, S L(2, \mathbb{R})\right), A_{l_{n+1}} \in S L(2, \mathbb{R})$ and $f_{l_{n+1}}^{\prime}(\theta) \in C_{\frac{1}{l_{n+2}}}^{\omega}\left(\mathbb{T}^{d}, s l(2, \mathbb{R})\right)$ such that

$$
\widetilde{B}_{l_{n}}(\theta+\alpha)^{-1} A_{l_{n}} e^{\overline{f_{l_{n}}}(\theta)} \widetilde{B}_{l_{n}}(\theta)=A_{l_{n+1}} e^{f_{l_{n+1}}^{\prime}(\theta)} .
$$

Let $B_{l_{n+1}}:=\widetilde{B}_{l_{n}} B_{l_{n}} \in C_{\frac{1}{l_{n+2}}}^{\omega}\left(2 \mathbb{T}^{d}, S L(2, \mathbb{R})\right)$, and note that whether or not the $\left(l_{n+1}\right)$-th step is in the resonant case, the following estimate holds:

$$
\left|f_{l_{n+1}}^{\prime}(\theta)\right|_{\frac{1}{l_{n+2}}}<\frac{1}{2} \varepsilon_{l_{n+1}^{\frac{5}{2}}} .
$$

We are going to analyze the structure of $A_{l_{n+1}}$ and estimate the norm of the conjugation $B_{l_{n+1}}$ in $\left(l_{n+1}\right)$-th step. Let $A_{l_{n+1}}:=e^{A_{l_{n+1}}^{\prime \prime}}$, and if $\left\|A_{l_{n+1}}^{\prime \prime}\right\|$ is sufficiently small, one can always find some unitary matrices $U \in S L(2, \mathbb{C})$ such that

$$
U^{-1} A_{l_{n+1}} U=\left(\begin{array}{cc}
e^{2 \pi i \rho_{n+1}} & c_{n+1} \\
0 & e^{-2 \pi i \rho_{n+1}}
\end{array}\right)
$$

with the estimate $\left|c_{n+1}\right| \leq 2\left\|A_{l_{n+1}}^{\prime \prime}\right\|$. Let us focus on the cocycle $\left(\alpha, A_{l_{n}} e^{\overline{f_{l_{n}}}(\theta)}\right)$ and we need to distinguish between two cases. 
(Resonant case). If the $\left(l_{n+1}\right)$-th step is obtained by resonant case, i.e., there exists $n_{l_{n+1}}^{*} \in$ $\mathbb{Z}^{d}$ such that

$$
\left\|2 \rho_{l_{n}}-\left\langle n_{l_{n+1}}^{*}, \alpha\right\rangle\right\|_{\mathbb{T}}<\varepsilon_{l_{n+1}}^{\sigma}, \quad 0<\left|n_{l_{n+1}}^{*}\right| \leq N_{l_{n+1}}:=\frac{2 \ln \left|\varepsilon_{l_{n+1}}\right|}{\frac{1}{l_{n+1}}-\frac{1}{l_{n+2}}},
$$

where $\left\{e^{2 \pi i \rho_{n}}, e^{-2 \pi i \rho_{n}}\right\}$ are two eigenvalues of $A_{l_{n}}$. Then by Proposition 3.1,

$$
\left|\widetilde{B}_{l_{n}}\right|_{\frac{1}{l_{n+2}}} \leq C_{3}(\gamma, \tau)\left(l_{n+1}\left|\ln \varepsilon_{l_{n+1}}\right|\right)^{\frac{\tau}{2}} \times \varepsilon_{l_{n+1}^{-\frac{2 \pi}{l_{n+1}-1}}} .
$$

We also have

$$
\left\|\widetilde{B}_{l_{n}}(\theta)\right\|_{0} \leq C_{3}\left(l_{n+1}\left|\ln \varepsilon_{l_{n+1}}\right|\right)^{\frac{\tau}{2}} .
$$

Hence,

$$
\begin{aligned}
\left|B_{l_{n+1}}\right| \frac{1}{l_{n+2}} & \leq C_{3}\left(l_{n+1}\left|\ln \varepsilon_{l_{n+1}}\right|\right)^{\frac{\tau}{2}} \times \varepsilon_{l_{n+1}}^{-\frac{2 \pi}{l_{n+1}-1}} \times\left(l_{n}\left|\ln \varepsilon_{l_{n}}\right|\right)^{\tau(\sigma+1)} \varepsilon_{l_{n}}^{-\xi} \\
& \leq\left(l_{n+1}\left|\ln \varepsilon_{l_{n+1}}\right|\right)^{\tau(\sigma+1)} \varepsilon_{l_{n+1}}^{-\xi} \\
\left\|B_{l_{n+1}}\right\|_{0} & \leq\left(l_{n+1}\left|\ln \varepsilon_{l_{n+1}}\right|\right)^{\tau(\sigma+1)} .
\end{aligned}
$$

Moreover one can get that $\left\|A_{l_{n+1}}^{\prime \prime}\right\| \leq 16 \varepsilon_{l_{n+1}}^{\sigma}$, which gives $\left|c_{n+1}\right| \leq 2\left\|A_{l_{n+1}}^{\prime \prime}\right\| \leq 32 \varepsilon_{l_{n+1}}^{\sigma}$. Combine with (3.8a), for any $\kappa \in\left[1, \frac{\sigma k}{8 \tau}\right)$, we have

$$
\left\|B_{l_{n+1}}\right\|_{0}^{K} \cdot\left|c_{n+1}\right| \leq 32\left|\ln \varepsilon_{l_{n+1}}\right|^{\frac{k \sigma}{4}} \cdot \varepsilon_{l_{n+1}}^{\frac{\sigma(1-\sigma)}{2}}<\infty .
$$

(Non-resonant case). If the $\left(l_{n+1}\right)$-th step is obtained by non-resonant case, we track back to the nearest resontant step, says the $\left(l_{m}\right)$-step. If such $m$ does not exist, we deduce that each step is in non-resonant case, thus

$$
\left\|A_{n+1}-A\right\| \leq\left\|A_{l_{1}}-A\right\|+\sum_{i=1}^{n}\left\|A_{l_{i+1}}-A_{l_{i}}\right\| \leq 8\|A\| \varepsilon_{l_{1}}
$$

and $\left|c_{n+1}\right| \leq\left\|A_{l_{n+1}}\right\| \leq 2\|A\|$. Since $B_{l_{n+1}}$ is close to identity by construction, it folllows that

$$
\left\|B_{l_{n+1}}\right\|_{0} \leq\left|B_{l_{n+1}}\right|_{\frac{1}{l_{n+1}}} \leq 2
$$

We deduce that

$$
\left\|B_{l_{n+1}}\right\|_{0}^{\kappa} \cdot\left|c_{n+1}\right| \leq 2^{\kappa+1}\|A\|<\infty .
$$

If such $m<n+1$ exists, let $A_{l_{m}}=e^{A_{l_{m}}^{\prime \prime}}$, then by (3.3) and (3.4), we have

$$
\left\|A_{l_{m}}^{\prime \prime}\right\| \leq 16 \varepsilon_{l_{m^{\prime}}}^{\sigma}, \quad\left\|A_{l_{m}}\right\| \leq 1+32 \varepsilon_{l_{l^{\prime}}}^{\sigma}, \quad\left|B_{l_{m}}\right|_{\frac{1}{l_{m+1}}} \leq\left(l_{m}\left|\ln \varepsilon_{l_{m}}\right|\right)^{\tau(\sigma+1)} \varepsilon_{l_{m}}^{-\xi} .
$$


Since each step is in non-resonant case from $\left(l_{m}\right)$-th step to $\left(l_{n+1}\right)$-th step, it deduces that

$$
\left\|A_{l_{n+1}}-A_{l_{m}}\right\| \leq 8\|A\| \varepsilon_{l_{m+1}}, \quad\left\|A_{l_{n+1}}\right\| \leq 2\|A\| .
$$

Moreover, (3.12) also implies

$$
\left|c_{n+1}\right| \leq 2\left\|A_{l_{n+1}}^{\prime \prime}\right\| \leq 64 \varepsilon_{l_{m}}^{\sigma} .
$$

Note that each conjugation is close to identity from $\left(l_{m}\right)$-step to $\left(l_{n+1}\right)$-step by construction in (3.5), thus one can get that

$$
\left\|B_{l_{n+1}}\right\|_{0} \leq\left|B_{l_{n+1}}\right|_{\frac{1}{l_{n+1}}} \leq 2\left\|B_{l_{m}}\right\|_{\frac{1}{l_{m+1}}} \leq\left(l_{n+1}\left|\ln \varepsilon_{l_{n+1}}\right|\right)^{\tau(\sigma+1)} \varepsilon_{l_{n+1}}^{-\tau} .
$$

Combine (3.13) with (3.14), it follows that

$$
\left\|B_{l_{n+1}}\right\|_{0}^{\kappa} \cdot\left|c_{n+1}\right| \leq 2^{\kappa+6}\left|\ln \varepsilon_{l_{m}}\right|^{\frac{k \sigma}{4}} \cdot \varepsilon_{l_{m}}^{\frac{\sigma(1-\sigma)}{2}}<\infty .
$$

This completes the proof of (A) by (3.6), (3.8a), (3.10) and (3.14). It also finishes the proof for (B) by (3.9), (3.11) and (3.15).

Remark 3.1. Theorem 3.1 has been proved in [10] essentially, however for the technical reason, we replace the estimate $\left\|B_{l_{j}}\right\|_{0} \leq \varepsilon_{l_{j}}^{-\frac{\sigma}{4}}$ in Proposition 3.2 of [10] by $\left\|B_{l_{j}}\right\|_{0} \leq$ $\left(l_{j}\left|\ln \varepsilon_{l_{j}}\right|\right)^{\tau(\sigma+1)}$, so that one can get $\left\|B_{l_{j}}\right\|_{0}^{\kappa} \cdot\left|c_{j}\right|<\infty$.

\subsection{Complex almost triangularization for $C^{k} \mathrm{Q}-\mathrm{P}$ cocycles}

In Theorem 3.1, one can see that the conjugation $B_{l_{j}}: 2 \mathbb{T}^{d} \rightarrow S L(2, \mathbb{R})$ is real, which results in $A_{l_{j}}$ being real. In fact, we can choose the complex conjugation $B_{l_{j}}: 2 \mathbb{T}^{d} \rightarrow$ $S L(2, \mathbb{C})$ to make $A_{l_{j}}$ complex almost triangularization provided that the cocycle is not uniformly hyperbolic.

Theorem 3.2. Suppose that all the conditions in Theorem 3.1 hold. Further assume that $\left(\alpha, A e^{f(\theta)}\right)$ is not uniformly hyperbolic. There exists $\varepsilon_{*}=\varepsilon_{*}(\gamma, \tau, d, k,\|A\|)$ such that if $\|f\|_{k} \leq \varepsilon_{*}$, then there exist $\widetilde{A}_{l_{j}} \in S L(2, \mathbb{C}), \widetilde{F}_{l_{j}} \in C^{k_{0}}\left(\mathbb{T}^{d}, S L(2, \mathbb{C})\right)$ with

$$
k_{0}=\left[\frac{k}{20}\right] \quad \text { and } \quad \Phi_{l_{j}}(\theta) \in C_{\frac{1}{l_{j+1}}}^{\omega}\left(2 \mathbb{T}^{d}, S L(2, \mathbb{C})\right) \text {, }
$$

such that

$$
\Phi_{l_{j}}(\theta+\alpha)^{-1} A e^{f(\theta)} \Phi_{l_{j}}(\theta)=\widetilde{A}_{l_{j}}+\widetilde{F}_{l_{j}}(\theta)
$$

where

$$
\widetilde{A}_{l_{j}}=\left(\begin{array}{cc}
e^{2 \pi i \rho_{j}} & c_{j} \\
0 & e^{-2 \pi i \rho_{j}}
\end{array}\right)
$$


with $\rho_{j} \in \mathbb{R}$ and $c_{j} \in \mathbb{C}$, also with estimates

$$
\left\|\widetilde{F}_{l_{j}}(\theta)\right\|_{0} \leq 2 \varepsilon_{l_{j^{\prime}}}^{\frac{1}{4}} \quad\left\|\Phi_{l_{j}}(\theta)\right\|_{0} \leq\left(l_{j}\left|\ln \varepsilon_{l_{j}}\right|\right)^{\tau(\sigma+1)}, \quad\left\|\Phi_{l_{j}}(\theta)\right\|_{0}^{\kappa} \cdot\left|c_{j}\right| \leq C,
$$

where $C$ is a constant defined in Theorem 3.1 and $\kappa \in\left[1, \frac{\sigma k}{8 \tau}\right)$.

Proof. Recall that in Theorem 3.1, we already have

$$
B_{l_{j}}(\theta+\alpha)^{-1} A e^{f_{l_{j}}(\theta)} B_{l_{j}}(\theta)=A_{l_{j}} e^{f_{l_{j}}^{\prime}(\theta)}, \quad \forall j \in \mathbb{Z} .
$$

Denote

$$
A_{l_{j}}+\widetilde{F}_{l_{j}}=A_{l_{j}} e^{f_{l_{j}}^{\prime}(\theta)}+B_{l_{j}}(\theta+\alpha)^{-1}\left(A e^{f}-A e^{f_{l_{j}}(\theta)}\right) B_{l_{j}}(\theta),
$$

then

$$
B_{l_{j}}(\theta+\alpha)^{-1} A e^{f_{l_{j}}(\theta)} B_{l_{j}}(\theta)=A_{l_{j}}+\widetilde{F}_{l_{j}}(\theta) .
$$

By the estimates of (A) in Theorem 3.1, we have

$$
\begin{aligned}
\left\|\widetilde{F}_{l_{j}}\right\|_{0} & \leq 2\left\|A_{l_{j}} f_{l_{j}}^{\prime}(\theta)\right\|_{0}+2\left\|B_{l_{j}}(\theta+\alpha)^{-1} A\left(f(\theta)-f_{l_{j}}(\theta)\right) B_{l_{j}}(\theta)\right\|_{0} \\
& \leq 2\|A\| \varepsilon_{l_{j}}^{\frac{5}{2}}+2\|A\|\left(l_{j}\left|\ln \varepsilon_{l_{j}}\right|\right)^{2 \tau(\sigma+1)} \times \frac{c}{(2\|A\|)^{D} l_{j}^{k-1}} \\
& \leq(2\|A\|)^{-1} \varepsilon_{l_{j^{\prime}}}^{2}
\end{aligned}
$$

where the second step uses the fact

$$
\sum_{i \geq j}\left\|f_{l_{i+1}}-f_{l_{i}}\right\| \leq \frac{c}{(2\|A\|)^{D} l_{j}^{k-1}}
$$

by (2.2).

Assume that $\left\{e^{2 \pi i \rho_{j}}, e^{-2 \pi i \rho_{j}}\right\}$ are two eigenvalues of $A_{l_{j}}$, then there exist unitary $U \in$ $S L(2, \mathbb{C})$, such that

$$
U^{-1} A_{l_{j}} U=\left(\begin{array}{cc}
e^{2 \pi i \rho_{j}} & c_{j} \\
0 & e^{-2 \pi i \rho_{j}}
\end{array}\right)
$$

with $\left|c_{j}\right| \leq\left\|A_{l_{j}}\right\| \leq 2\|A\|$, where $\rho_{j} \in i \mathbb{R} \cup \mathbb{R}$ and $c_{j} \in \mathbb{C}$. In the following, we need to rule out that $i \rho_{j} \in \mathbb{R} \backslash\{0\}$. Suppose that $\lambda_{j}=i \rho_{j} \in \mathbb{R} \backslash\{0\}$. If $2 \pi\left|\rho_{j}\right|>\varepsilon_{l_{j}}^{\frac{1}{4}}$ let $P:=$ $\operatorname{diag}\left\{\|2 A\|^{\frac{1}{2}} \varepsilon_{l_{j}}^{-\frac{1}{2}},\|2 A\|^{-\frac{1}{2}} \varepsilon_{l_{j}}^{\frac{1}{2}}\right\}$, then we have

$$
P^{-1} U^{-1}\left(A_{l_{j}}+\widetilde{F}_{l_{j}}\right) U P=\left(\begin{array}{cc}
e^{2 \pi \lambda_{j}} & 0 \\
0 & e^{-2 \pi \lambda_{j}}
\end{array}\right)+F(\theta)
$$

with $\|F\|_{0} \leq 2 \varepsilon_{l_{j}}$. We rewrite

$$
\left(\begin{array}{cc}
e^{2 \pi \lambda_{j}} & 0 \\
0 & e^{-2 \pi \lambda_{j}}
\end{array}\right)+F(\theta)=\left(\begin{array}{cc}
e^{2 \pi \lambda_{j}} & 0 \\
0 & e^{-2 \pi \lambda_{j}}
\end{array}\right) e^{\widetilde{f}(\theta)}
$$


with $\|\widetilde{f}(\theta)\|_{0} \leq 4\|A\| \varepsilon_{l_{j}}$.

By Lemma 2.2 and Corollary 3.1 of [17], one can conjugate (3.17) to

$$
\left(\begin{array}{cc}
e^{2 \pi \lambda_{j}} & 0 \\
0 & e^{-2 \pi \lambda_{j}}
\end{array}\right)\left(\begin{array}{cc}
e^{\tilde{f}^{r e}}(\theta) & 0 \\
0 & e^{-\widetilde{f}^{r e}(\theta)}
\end{array}\right)=\left(\begin{array}{cc}
e^{2 \pi \lambda_{j}} e^{\tilde{f}^{r e}(\theta)} & 0 \\
0 & e^{-2 \pi \lambda_{j}} e^{-\widetilde{f}^{r e}(\theta)}
\end{array}\right)
$$

with $\left\|\tilde{f}^{r e}(\theta)\right\|_{0} \leq 8\|A\| \varepsilon_{l_{j}}$, thus $\left(\alpha, A e^{f(\theta)}\right)$ is uniformly hyperbolic, which contradicts to the assumption. Hence we only need to consider

$$
2 \pi\left|\rho_{j}\right| \leq \varepsilon_{l_{j}}^{\frac{1}{4}}
$$

In this case, we put $\rho_{j}$ into the perturbation so that the new perturbation satisfies

$$
\left\|\widetilde{F}_{l_{j}}\right\|_{0} \leq 2 \varepsilon_{l_{j}}^{\frac{1}{4}} \text { and } \quad A_{l_{j}}=\left(\begin{array}{cc}
1 & c_{j} \\
0 & 1
\end{array}\right) .
$$

Denote

$$
\Phi_{l_{j}}(\theta)=B_{l_{j}}(\theta) U \in C_{\frac{1}{l_{j+1}}}^{\omega}\left(2 \mathbb{T}^{d}, S L(2, \mathbb{C})\right)
$$

we always have

$$
\Phi_{l_{j}}(\theta+\alpha)^{-1} A e^{f(\theta)} \Phi_{l_{j}}(\theta)=\left(\begin{array}{cc}
e^{2 \pi i \rho_{j}} & c_{j} \\
0 & e^{-2 \pi i \rho_{j}}
\end{array}\right)+\widetilde{F}_{l_{j}}(\theta)
$$

with $\rho_{j} \in \mathbb{R}, c_{j} \in \mathbb{C}$ and $\left\|\widetilde{F}_{l_{j}}\right\|_{0} \leq 2 \varepsilon_{l_{j}}^{\frac{1}{4}}$. Since $U$ is unitary, by Theorem 3.1(A), (3.16) holds.

\section{Sharp Hölder continuity of the spectral measure}

Consider the following discrete $C^{k} \mathrm{Q}-\mathrm{P}$ Schrödinger operators:

$$
\left(H_{V, \alpha, \theta} u\right)_{n}=u_{n+1}+u_{n-1}+V(\theta+n \alpha) u_{n}, \quad \forall n \in \mathbb{Z},
$$

where $\alpha \in \mathrm{DC}_{d}(\gamma, \tau)$ and $V \in C^{k}\left(\mathbb{T}^{d}, \mathbb{R}\right)$. Let $\mu_{V, \alpha, \theta}$ be the spectral measure of $H_{V, \alpha, \theta}$. Based on the dynamical estimates of corresponding Schrödinger cocycles, we are able to show the $\frac{1}{2}$-Hölder continuity of $\mu_{V, \alpha, \theta}$.

Let $A(\theta):=S_{E}^{V}(\theta)$ and for any $n \geq 1$, we define

$$
P_{n}(\theta)=\sum_{s=1}^{n} A_{2 s-1}^{*}(\theta+\alpha) A_{2 s-1}(\theta+\alpha),
$$

where $A_{s}(\theta)=A(\theta+(s-1) \alpha) \cdots A(\theta+\alpha) A(\theta) . P_{n}$ is an increasing family of positive self-adjoint operators. $\left\|P_{n}\right\|$ is unbounded since $\operatorname{tr} P_{n} \geq 2 n$. Moreover, $\operatorname{det} P_{n}$ is also unbounded. For simplicity, we will use the notation $a \approx b$ which denotes that there exist some constants $C>0$ such that $C^{-1} a \leq b \leq C a$ and also use the notation $a \lesssim b$ which denotes $a \leq C b$ for some constants $C>0$. 
Theorem 4.1. Let $\alpha \in \mathrm{DC}_{d}(\gamma, \tau), V \in C^{k}\left(\mathbb{T}^{d}, \mathbb{R}\right)$ with $k \geq 5 D \tau$ and $D$ is a numerical constant. There exists $\tilde{\varepsilon}=\tilde{\varepsilon}(\gamma, \tau, k)$ such that if $\|V\|_{k} \leq \tilde{\varepsilon}$, then for any $f \in \ell^{2}(\mathbb{Z}) \cap \ell^{1}(\mathbb{Z})$,

$$
\mu_{V, \alpha, \theta}^{f}(J) \leq D_{0}\|f\|_{\ell^{1}}^{2} \cdot|J|^{\frac{1}{2}},
$$

for all intervals $J$ and all $\theta$, where $D_{0}=D_{0}(V, \alpha)>0$.

Proof. Since the spectral measure $\mu_{V, \alpha, \theta}$ vanishes on $\mathbb{R} \backslash \Sigma_{V, \alpha}$, we only need to consider the case $E \in \Sigma_{V, \alpha}$.

Rewrite the Schrödinger cocycle $\left(\alpha, S_{E}^{V}\right)$ as $\left(\alpha, A_{E} e^{f(\theta)}\right)$, where

$$
A_{E}=\left(\begin{array}{cc}
E & -1 \\
1 & 0
\end{array}\right), \quad f(\theta)=\ln \left(I d+A_{E}^{-1} F(\theta)\right), \quad F(\theta)=\left(\begin{array}{cc}
-V(\theta+n \alpha) & 0 \\
0 & 0
\end{array}\right) .
$$

By the assumption on $\|V\|_{k}$ and Theorem 3.2, for $\kappa \in\left[1, \frac{\sigma k}{8 \tau}\right)$, there exist $\Phi_{l_{j}} \in$ $C^{k}\left(2 \mathbb{T}^{d}, S L(2, \mathbb{C})\right)$ with $\left\|\Phi_{l_{j}}\right\|_{0} \leq\left(l_{j}\left|\ln \varepsilon_{l_{j}}\right|\right)^{\tau(\sigma+1)}$ and $\beta_{i} \in C^{k_{0}}\left(\mathbb{T}^{d}, \mathbb{R}\right), i=1,2,3,4$ with $k_{0}=\left[\frac{k}{20}\right]$ such that

$$
\Phi_{l_{j}}(\theta+\alpha)^{-1} S_{E}^{V}(\theta) \Phi_{l_{j}}(\theta)=T_{l_{j}}+\left(\begin{array}{ll}
\beta_{1}(\theta) & \beta_{2}(\theta) \\
\beta_{3}(\theta) & \beta_{4}(\theta)
\end{array}\right)
$$

where

$$
T_{l_{j}}=\left(\begin{array}{cc}
e^{2 \pi i \rho_{j}} & c_{j} \\
0 & e^{-2 \pi i \rho_{j}}
\end{array}\right)
$$

with $\rho_{j} \in \mathbb{R}$ and $c_{j} \in \mathbb{C}$, also with estimates

$$
\left\|\Phi_{l_{j}}\right\|_{0}^{\kappa} \cdot\left|c_{j}\right| \leq C, \quad\left\|\beta_{i}\right\|_{0} \leq 2 \varepsilon_{l_{j^{\prime}}}^{\frac{1}{4}} \quad i=1,2,3,4
$$

For simplicity of notations, in the following $\Phi_{l_{j}}, T_{l_{j}}, \rho_{j}$ are written as $\Phi, T, \rho$ respectively. Let $\widetilde{T}(\theta)=\Phi(\theta+\alpha)^{-1} S_{E}^{V}(\theta) \Phi(\theta)$, then we have

$$
\|\widetilde{T}-T\|_{0} \leq 2 \varepsilon_{l_{j}}^{\frac{1}{4}}
$$

We need to compare the dynamics between $\left(\alpha, S_{E}^{V}\right)$ and $(\alpha, T)$. For this purpose, let $X=$ $\sum_{j=1}^{n} T_{2 j-1}^{*} T_{2 j-1}$ and $\widetilde{X}(\theta)=\sum_{j=1}^{n} \widetilde{T}_{2 j-1}^{*}(\theta) \widetilde{T}_{2 j-1}(\theta)$. The following estimates on $\|X\|_{0}$ and $\left\|X^{-1}\right\|_{0}^{-1}$ are crucial.

Lemma 4.1 (Lemma 4.3 of [5]). Let

$$
K(\theta)=\left(\begin{array}{cc}
e^{2 \pi i \rho} & t(\theta) \\
0 & e^{-2 \pi i \rho}
\end{array}\right),
$$

where $t(\theta)=\widehat{t}(r) e^{2 \pi i r \theta}$. Let $G=\sum_{j=1}^{n} K_{2 j-1}^{*} K_{2 j-1}$, then

$$
\begin{aligned}
& \|G\|_{0} \approx n\left(1+|\widehat{t}(r)|^{2} \min \left\{n^{2},\|2 \rho-\langle r, \alpha\rangle\|_{\mathbb{T}}^{-2}\right\}\right), \\
& \left\|G^{-1}\right\|_{0}^{-1} \approx n .
\end{aligned}
$$


Lemma 4.2 (Lemma 4.4 of [5]). Let $G$ and $\widehat{t}(r)$ be as above. Define $\widetilde{G}=\sum_{j=1}^{n} \widetilde{K}_{2 j-1}^{*} \widetilde{K}_{2 j-1}$. Then there exists $D_{1}>0$ such that if

$$
\|\widetilde{K}-K\|_{0} \leq D_{1} n^{-2}(1+2 n|\widehat{t}(r)|)^{-2},
$$

we have $\|\widetilde{G}-G\|_{0} \leq 1$.

Apply Lemma 4.1, one can get that

$$
\|X\|_{0} \approx n\left(1+\left|c_{j}\right|^{2} \min \left\{n^{2},\|2 \rho\|_{\mathbb{T}}^{-2}\right\}\right), \quad\left\|X^{-1}\right\|_{0}^{-1} \approx n .
$$

Let $n^{+}$be maximal such that $\|\widetilde{X}-X\|_{0} \leq 1$ for $1 \leq n<n^{+}$. So by Lemma 4.2 and (4.3), one can get that

$$
2 \varepsilon_{l_{j}}^{\frac{1}{4}} \geq D_{1}\left(n^{+}\right)^{-2}\left(1+2 n^{+}\left|c_{j}\right|\right)^{-2} \gtrsim\left(n^{+}\right)^{-4} .
$$

It follows that

$$
n^{+} \gtrsim \varepsilon_{l_{j}}^{\frac{1}{16}}
$$

Since $\|\widetilde{X}\|_{0} \leq\|X\|+1$ and $\left\|\widetilde{X}^{-1}\right\| \geq\left\|X^{-1}\right\|_{0}-1$ for $1 \leq n<n^{+}$, also we notice that

$$
\begin{aligned}
& \|P\|_{n} \leq\|\Phi(\theta)\|_{0}^{4} \cdot\|\widetilde{X}(\theta+\alpha)\|_{0}, \\
& \left\|P_{n}^{-1}\right\|_{0} \geq\|\Phi(\theta)\|_{0}^{-4} \cdot\left\|\widetilde{X}(\theta+\alpha)^{-1}\right\|_{0}^{-1} .
\end{aligned}
$$

(4.4) implies

$$
\begin{aligned}
& \left\|P_{n}\right\|_{0} \leq D_{2} n\left(1+\left|c_{j}\right|^{2} n^{2}\right)\|\Phi\|_{0}^{4} \\
& \left\|P_{n}^{-1}\right\|_{0}^{-1} \geq D_{3} n\|\Phi\|_{0}^{-4} .
\end{aligned}
$$

Hence by direct calculation,

$$
\frac{\left\|P_{n}\right\|_{0}}{\left\|P_{n}^{-1}\right\|_{0}^{-3}} \leq D_{4}\left|c_{j}\right|^{2} \cdot\|\Phi\|_{0}^{16}+D_{4} \frac{1}{n^{2}}\|\Phi\|_{0}^{16}
$$

From Theorem 3.2, we know that $\|\Phi\|_{0}^{8} \cdot\left|c_{j}\right|<\infty$ and $\|\Phi\|_{0} \leq \varepsilon_{l_{j}}^{-\frac{\sigma}{30}}$, then

$$
\left\|P_{n}\right\|_{0} \lesssim\left\|P_{n}^{-1}\right\|_{0}^{-3}, \quad \forall n \in\left(n^{-}, n^{+}\right)
$$

where

$$
n^{-}:=\varepsilon_{l_{j}}^{-\frac{8 \sigma}{30}}
$$

Denote the interval $I_{j}:=\left[D_{5} l_{j}^{\frac{k}{150}}, D_{6} l_{j}^{\frac{k}{64}}\right], j \in \mathbb{Z}^{+}$, then by (4.5) and (4.6), for any $n \in I_{j}$, we have $n^{-}<n<n^{+}$. Since $I_{j} \cap I_{j+1} \neq \varnothing, \forall j \in \mathbb{Z}^{+}$, thus $\cup_{j \geq 1} I_{j}$ cover all the $n$ tending to 
infinity. Hence $\left\|P_{n}\right\|_{0} \lesssim\left\|P_{n}^{-1}\right\|_{0}^{-3}$ for any $n \geq D_{5} l_{1}^{\frac{k}{150}}$. On the other hand, the number of $n$ satisfying $n<D_{5} l_{1}^{\frac{k}{150}}$ is finite, then

$$
\sup _{n<D_{5} l_{1}^{\frac{k}{150}}} \frac{\left\|P_{n}\right\|_{0}}{\left\|P_{n}^{-1}\right\|_{0}^{-3}}<\infty .
$$

Thus

$$
\left\|P_{n}\right\| \lesssim\left\|P_{n}^{-1}\right\|^{-3}, \quad \forall n \in \mathbb{Z}^{+} .
$$

Lemma 4.3 (Lemma 4.2 of [5]). Let $\epsilon_{n}=\frac{1}{2 \sqrt{\operatorname{det} P_{n}}}$, then

$$
D_{6}^{-1}<\frac{\psi\left(m^{+}\left(E+i \epsilon_{n}\right)\right)}{2 \epsilon_{n}\left\|P_{n}\right\|_{0}}<D_{6}
$$

where $D_{6}>0$ is a constant and $\psi\left(m^{+}\right)$is defined in Lemma 2.1.

For any bounded potential and any solution $u$ satisfying (4.1), we have

$$
\|u\|_{L+1} \lesssim\|u\|_{L}, \quad \text { where }\|u\|_{L}=\left(\sum_{j=1}^{L}\left|u_{j}\right|^{2}\right)^{\frac{1}{2}} .
$$

In particular, for the solution $u^{\beta}$ with $u_{0}^{\beta} \cos \beta+u_{1}^{\beta} \sin \beta=0$ and $\left|u_{0}^{\beta}\right|^{2}+\left|u_{1}^{\beta}\right|^{2}=1$, we have

$$
\operatorname{det} P_{n}=\inf _{\beta}\left\|u^{\beta}\right\|_{L}^{2}\left\|u^{\beta+\pi / 2}\right\|_{L}^{2} \text {. }
$$

By (4.7) and $P_{n}: \mathbb{T}^{d} \rightarrow \operatorname{gl}(2, \mathbb{R})$, we have

$$
\left\|P_{n}\right\|_{0}=\operatorname{det} P_{n}\left\|P_{n}^{-1}\right\|_{0} \lesssim \epsilon_{n}^{-2}\left\|P_{n}\right\|^{-\frac{1}{3}},
$$

where $\epsilon_{n}$ is defined in Lemma 4.3. Thus $\left\|P_{n}\right\|_{0} \lesssim \epsilon_{n}^{-\frac{3}{2}}$. According to Lemma 4.3, we deduce that

$$
\psi\left(m^{+}\left(E+i \epsilon_{n}\right)\right) \lesssim \epsilon_{n}\left\|P_{n}\right\|_{0} \lesssim \epsilon_{n}^{-\frac{1}{2}} .
$$

Since $\lim _{n \rightarrow \infty} \epsilon_{n}=0$, and we also have $\epsilon_{n} \lesssim \epsilon_{n+1}$ by (4.8), we only need to consider the case of fixing $\epsilon_{n}=\epsilon$. Combine (2.1) with Lemma 2.1, we have

$$
\mu_{V, \alpha, \theta}(E-\epsilon, E+\epsilon) \leq 2 \epsilon \Im M(E+i \epsilon) \lesssim \epsilon^{\frac{1}{2}} .
$$

Since $\mu_{V, \alpha, \theta}=0$ on $\mathbb{R} \backslash \Sigma_{V, \alpha}$, then there exists $D_{0}=D_{0}(V, \alpha)>0$ such that

$$
\mu_{V, \alpha, \theta}(J) \leq D_{0}|J|^{\frac{1}{2}}, \quad \forall J \subset \mathbb{R} .
$$


Let $\sigma: \ell^{2}(\mathbb{Z}) \rightarrow \ell^{2}(\mathbb{Z})$ be the shift $f(i+1)=\sigma f(i)$. Then $\sigma H_{V, \alpha, \theta} \sigma^{-1}=H_{V, \alpha, \theta+\alpha}$. Thus $\mu_{\theta+\alpha}^{\sigma f}=\mu_{\theta}^{f}$ and $\mu_{\theta}^{e_{k}}=\mu_{\theta+k \alpha}^{e_{0}} \leq \mu_{\theta+k \alpha}$. Let $\mathbb{E}(J)$ be the spectral projection of $H_{V, \alpha, \theta}$ on $J$, then

$$
\begin{aligned}
\mu_{V, \alpha, \theta}^{f}(J) & =\langle\mathbb{E}(J) f, f\rangle=\left\|\mathbb{E}(J) \sum_{k} f(k) e_{k}\right\|^{2} \leq\left(\sum_{k}|f(k)| \cdot\left\|\mathbb{E}(J) e_{k}\right\|\right)^{2} \\
& =\left(\sum_{k}|f(k)| \mu_{V, \alpha, \theta}^{e_{k}}(J)^{\frac{1}{2}}\right)^{2} \leq\left(\sum_{k}|f(k)| \mu_{V, \alpha, \theta+k \alpha}(J)^{\frac{1}{2}}\right)^{2} \\
& \leq D_{0}\|f\|_{\ell^{1}}^{2} \cdot|J|^{\frac{1}{2}} .
\end{aligned}
$$

Thus, we complete the proof.

\section{Acknowledgements}

The authors want to thank Jiangong You and Qi Zhou for useful discussions. Mei Sun was supported by National Nature Science Foundation of China grant (No. 71774070).

\section{References}

[1] A. Avila, Global theory of one-frequency Schrödinger operators, Acta. Math., 215 (2015), $1-54$.

[2] A. Avila, The absolutely continuous spectrum of the almost Mathieu operator, arXiv: 0810.2965.

[3] A. Avila, KAM, Lyapunov exponents and the spectral dichotomy for one-frequency Schrödinger operators, preparation.

[4] A. Avila, and S. Jitomirskaya, Almost localization and almost reducibility, J. Euro. Math. Soc., 12 (2010), 93-131.

[5] A. Avila, and S. Jitomirskaya, Hölder continuity of absolutely continuous spectral measures for one-frequency Schrödinger operators, Commun. Math. Phys., 301 (2011), 563-581.

[6] J. Avron, and B. Simon, Almost periodic Schrödinger operators II, the integrated density of states, Duke. Math. J., 506 (1983), 369-390.

[7] J. Bourgain, Hölder regularity of integrated density of states for the almost Mathieu operator in a perturbative regime, Lett. Math. Phys., 51 (2000), 83-118.

[8] J. Bourgain, On the spectrum of lattice Schrödinger operators with deterministic potential. II, J. Anal. Math., 88 (2002), 221-254.

[9] A. Cai, and L. Ge, Reducibility of finitely differentiable quasi-periodic cocycles and its spectral applications, arXiv:1712.09041.

[10] A. Cai, C. Chavaudret, J. You, and Q. Zhou, Sharp Hölder continuity of the Lyapunov exponent of finitely differentiable quasi-periodic cocycles, Math. Z., 291 (2019), 931-958.

[11] D. Damanik, Schrödinger operators with dynamically defined potentials, Erg. Theory. Dyn. Syst., 37 (2017), 1681-1764.

[12] D. Damanik, R. Killip, and D. Lenz, Uniform spectral properties of one-dimensional quasicrystals. iii, $\alpha$-continuity, Commun. Math. Phys., 212 (2000), 191-204.

[13] L. H. Eliasson, Floquet solutions for the 1-dimensional quasi-periodic Schrödinger equation, Commun. Math. Phys., 146 (1992), 447-482. 
[14] M. Goldstein, and W. Schlag, Hölder continuity of the integrated density of states for quasi-periodic Schrödinger equations and averages of shifts of subharmonic functions, Ann. Math., 154 (2001), 155-203.

[15] M. Goldstein, and W. Schlag, Fine properties of the integrated density of states and a quantitative separation property of the Dirichlet eigenvalues, Geom. Funct. Anal., 18 (2008), 755869.

[16] S. Hadj Amor, Hölder continuity of the rotation number for quasi-periodic cocycles in $\mathrm{SL}(2, \mathbb{R})$, Commun. Math. Phys., 187 (2009), 565-588.

[17] X. Hou, and J. You, Almost reducibility and non-perturbative reducibility of quasi-periodic linear systems, Invent. math., 190 (2012), 209-260.

[18] S. Jitomirskaya, Almost Everything About the Almost Mathieu Operator, II, Proceedings of XI International Congress of Mathematical Physics, Int. Press, (1995), 373-382.

[19] R. Johnson, Exponential dichotomy, rotation number, and linear differential operators with bounded coefficients, J. Differential Equations, 61 (1986), 54-78.

[20] M. Leguil, J. You, Z. Zhao, and Q. Zhou, Asymptotics of spectral gaps of quasi-periodic Schrödinger operators, arXiv:1712.04700.

[21] W. Liu, and X. Yuan, Hölder continuity of the spectral measures for one-dimensional Schrödinger operator in exponential regime, J. Math. Phys., 56 (2015), 012701.

[22] J. You, and S. Zhang, Hölder continuity of the Lyapunov exponent for analytic quasiperiodic Schrödinger cocycle with weak Liouville frequency, Erg. Theory. Dyn. Syst., 34 (2014), 13951408.

[23] E. Zehnder, Generalized implicit function theorems with application to some small divisor problems, I, Commun. Pure. Math., XXVIII (1975), 91-140.

[24] X. Zhao, Hölder continuity of absolutely continuous spectral measure for multi-frequency Schrödinger operators, J. Funct. Anal., (2020), https ://doi .org/10.1016/j .jfa. 2020. 108508. 